\title{
Evapotranspiração e coeficiente de cultivo do tomate caqui cultivado em ambiente protegido ${ }^{1}$
}

\author{
$\overline{\text { Ligia S. Reis }{ }^{2} \text {, José L. de Souza }{ }^{3} \text { \& Carlos A. V. de Azevedo }}$
}

\begin{abstract}
RESUMO
Os parâmetros aerodinâmicos de uma cultura cultivada em ambiente protegido podem ser considerados dependentes do nível de radiação global, temperatura do ar e umidade do ar, com base em leis exponenciais. Assim sendo se propôs com este trabalho, estimar a evapotranspiração e o coeficiente de cultivo da cultura do tomate caqui em ambiente protegido, sob irrigação por gotejamento, utilizando-se o modelo de Penman-Monteith. Os parâmetros aerodinâmicos foram medidos com sensores conectados à estação automática instalada dentro do ambiente protegido. A evapotranspiração da cultura (ETc) foi determinada experimentalmente por meio de lisímetros de drenagem e a umidade do solo foi medida através de sensores instalados a uma profundidade de $20 \mathrm{~cm}$. O desempenho do modelo de Penman-Monteith foi comparado aos valores decendiais do balanço hídrico nos lisímetros; já a evapotranspiração de referência foi calculada com dados externos e utilizada para o cálculo do Kc da cultura; enfim, os resultados indicaram que o modelo de Penman-Monteith subestima os valores de evapotranspiração encontrados pelo balanço hídrico nos lisímetros.
\end{abstract}

Palavras-chave: manejo de água, lisimetria, Penman-Monteith

\section{Evapotranspiration and crop coefficient of Kaki tomato cultivated in greenhouse}

\begin{abstract}
The aerodynamic parameters of a crop cultivated in greenhouse can be considered dependent upon the level of global radiation, air temperature and relative humidity, based on exponential laws. Consequently, this work intends to estimate the evapotranspiration and the crop coefficient of Kaki tomato in greenhouse, under drip irrigation, using the PenmanMonteith model. The aerodynamic parameters were measured with sensors connected to the automatic station installed inside the greenhouse. The ETc was determined experimentally through drainage lysimeters and the soil water content was measured through sensors installed at a depth of $20 \mathrm{~cm}$. The performance of the Penman-Monteith model was compared to decennial values of the water balance in the lysimeters, while the reference evapotranspiration was calculated with external data and used for calculation of crop Kc. The results indicated that the Penman-Monteith model underestimates the evapotranspiration values found by the water balance in the lysimeters.
\end{abstract}

Key words: water management, lisimeter, Penman-Monteith

\footnotetext{
1 Parte da Tese de Doutorado do primeiro autor apresentada à UFCG

${ }^{2}$ CECA/UFAL, Campus Delza Gitaí, BR 101 - Norte, km 14, Rio Largo, AL. E-mail: Isr@fapeal.br

${ }^{3}$ CECA/UFAL, A. C. Simões, BR 104 - Norte, km 97, Tabuleiro dos Martins, CEP 57072-970, Maceió, AL. E-mail: jls@ccen.ufal.br

${ }^{4}$ DEAg/UFCG, Av. Aprígio Veloso, 882, Bairro Universitário, CEP 58429-140, Campina Grande, PB. Fone: 83 3310-1056. E-mail: cazevedo@deag.ufcg.edu.br
} 


\section{INTRODUÇÃO}

O cultivo de culturas em estufa plástica tem, como objetivo, a obtenção de maior produtividade com melhor qualidade do produto final durante o ano. Na estufa, o plástico utilizado como cobertura visa à proteção das culturas contra as adversidades agroclimáticas, técnica que vem sendo amplamente utilizada em vários países (Boulard \& Wang, 2000; Kirda et al., 2003; Orgaz et al., 2005). No Brasil, a utilização de ambiente protegido, principalmente para a produção de plantas ornamentais e hortícolas, se vem alastrando devido à maior proteção quanto aos fenômenos climáticos, como: geadas, excesso de chuvas, diminuição da temperatura noturna, proteção do solo contra a lixiviação e redução dos custos com fertilizantes e defensivos, enquanto as colheitas nesses ambientes excedem as que se obtêm a céu aberto (Oliveira, 1995; Fayad et al., 2001; Cunha et al., 2002; Carrijo et al., 2004).

Práticas como a utilização de "mulching" sobre o solo nas linhas de cultivo e a irrigação por gotejamento controlado, recomendadas para cultivos em estufa, diminuem a evaporação da água na superfície do solo e contribuem para diminuir ainda mais o consumo d'água e a freqüência de irrigação das culturas nesse ambiente, sobretudo quando a parte aérea das plantas não recobre toda a superfície. A água é o fator mais importante a ser considerado para maximizar a produção e melhorar a qualidade dos produtos agrícolas; entretanto, sua eficiência consiste em oferecer a quantidade certa no momento adequado requerido pela planta. Portanto, os valores da evapotranspiração máxima e do coeficiente de cultura (Kc) para as condições do campo, como os determinados para tomateiro (Lima et al., 1994), melancia (Miranda et al., 2004) ou aqueles indicados por Doorenbos \& Pruitt (1976) para diferentes hortaliças, não podem ser utilizados diretamente para o ambiente parcialmente modificado de uma estufa plástica.

Várias correlações entre transpiração e os parâmetros climáticos têm sido determinadas experimentalmente. Joillete \& Bailey (1992), Dalsasso et al. (1997) e Valandro et al. (1999) avaliaram a transpiração do tomateiro quando ficou evidente a dificuldade de se determinar um modelo para estimativa do consumo d'água pelas plantas no cultivo outono-inverno, em Santa Maria; esta dificuldade advém do fato de que existem entradas de frentes frias.

O tomate (Lycopersicum esculentum, Mill) é uma das hortaliças mais significativas cultivadas no Brasil (Luz et al., 2007) por se tratar de uma das principais culturas exploradas em estufa plástica, resultando em frutos de excelente qualidade comercial garantindo, assim, um lucro maior. Vários trabalhos vêm sendo apresentados, comprovando o aumento na produção de algumas culturas em função de alterações micrometeorológicas promovidas pelo uso de estufa com cobertura plástica (Buriol et al., 1993; Heldwein et al., 2004, Cunha et al., 2002; Blanco \& Folegatti, 2003).

As vantagens promovidas pelo cultivo em ambiente protegido quando comparado com cultivo no campo, são inúmeras, como a redução na incidência de radiação solar (Souza et al., 1997) e na velocidade do vento e, conseqüen- temente, redução da evapotranspiração; mesmo assim, é pouco o conhecimento sobre as interações do desenvolvimento e produção das culturas com condições microclimáticas nesse ambiente. Um dos fatores que mais recebem influência dos elementos que compõem a casa de vegetação é a radiação solar, que é a fonte básica para evaporação de água de superfície.

É muito difícil conseguir separar a ação de cada um dos parâmetros meteorológicos na evapotranspiração, pois se sabe que eles agem ao mesmo tempo. Em geral, pode-se afirmar que, quanto maior a disponibilidade de energia solar, da temperatura do ar, da velocidade do vento e menor for a umidade relativa, maior também será a taxa de evapotranspiração (Cunha \& Escobedo, 2003). Embora a temperatura e a umidade relativa do ar possam, em alguns momentos, atingir valores superiores no interior do ambiente protegido, com estreita dependência das condições meteorológicas, comumente a evapotranspiração no interior do ambiente protegido fica em torno de $60-80 \%$ da verificada no exterior (Farias et al., 1993). Tais reduções induzem à necessidade de estudos detalhados dos fenômenos microclimáticos possibilitando estimar a evapotranspiração.

Vários são os métodos para estimativa da evapotranspiração da cultura (ETc), a exemplo da equação de PenmanMonteith, porém o uso desta equação está condicionado a uma estimativa correta da resistência do dossel (Rana \& Katerji, 2000). Inúmeros estudos foram realizados com a equação Penman-Monteith, a exemplo de Ortega-Farías \& Cuenca $(1996,1998)$ e Ortega-Farías et al. (2000), que propuseram o uso do método a fim de se determinar o coeficiente de cultivo em diferentes condições edafoclimáticas.

Considerando o exposto, o presente trabalho tem, como objetivo, estimar a evapotranspiração e o coeficiente de cultivo do tomateiro em ambiente protegido através da equação de Penman-Monteith, comparando essas estimativas com medidas de lisímetros de drenagem.

\section{MATERIAL E MÉTODOS}

Este trabalho foi conduzido no Campus Delza Gitaí, no Centro de Ciências Agrárias da Universidade Federal de Alagoas, no Município de Rio Largo (09² 28' S, 35 49’ W e $127 \mathrm{~m}$ de altitude) cujo clima é quente úmido, com moderada deficiência de água no verão (S), e grande excesso no inverno (W), segundo a classificação de Thorntwaite-Mather. Nesta região ocorrem chuvas entre março a agosto, que representam $78 \%$ da precipitação total anual; o período seco acontece de setembro a fevereiro e a média anual é de $1818 \mathrm{~mm}$ (Souza et al., 2005).

A área de ambiente protegido era de 17 x $6,5 \mathrm{~m}$, o polietileno usado foi o de $120 \mu \mathrm{m}$ de espessura na cobertura e as laterais de sombrite a $50 \%$; a adubação foi realizada de acordo com a análise do solo, o transplantio ocorreu no dia 2 de junho de 2005, com espaçamento de 0,4 x 1,0 m, em 6 fileiras de plantas. Quatro lisímetros com dimensões 1,0 x 1,0 x $0,7 \mathrm{~m}$ foram instalados no centro da estufa, com duas plantas cada um. 
A irrigação se deu por gotejamento, efetuada com mangueiras espaçadas $1 \mathrm{~m}$ entre si e $0,40 \mathrm{~m}$ entre emissores e vazão média de $8 \mathrm{~L} \mathrm{~h}^{-1}$ por emissor. Determinou-se a lâmina de irrigação aplicada com base na evapotranspiração de referência da área experimental, que foi de $3 \mathrm{~mm} \mathrm{~d}^{-1}$, e um turno de rega fixo de dois dias, levando-se em consideração a fase de desenvolvimento da cultura.

Obtiveram-se as observações meteorológicas com sensores instalados no centro da área cultivada acoplados a um sistema automático de aquisição de dados, modelo CR10X da Campbell Scientific, programado para fazer medições a cada dez segundos e armazenar médias a cada dez minutos, dos elementos temperatura do ar (T) e umidade do ar (UR), (modelo HPM 45C, a $2 \mathrm{~m}$ de altura), velocidade do vento (VV) medida a 2,0 m do dossel vegetativo, piranômetro (CM3, Kipp Zone) para medida de radiação solar global (Rg).

A umidade do solo foi monitorada por dois sensores de umidade volumétrica (CS615 Water Content Reflectometers) da Campbell (um instalado no lisímetro cultivado e outro no lisímetro sem plantas), obtendo-se a umidade do solo média entre a superfície e $30 \mathrm{~cm}$ de profundidade por relação entre o período $\mathrm{P}$, da onda de sensor e a umidade de volume real $(\theta)$, cuja calibração foi feita com um modelo encontrado para o solo da área de estudo $\left(\theta=0,13-0,00689 \mathrm{P}+0,0003631 \mathrm{P}^{2}\right)$ e ajustado por um fator obtido entre as densidades do solo, em condições naturais e o solo do lisímetro.

Para o cálculo da evapotranspiração diária utilizou-se a derivação da equação de Penman-Monteith, apresentada abaixo (Monteith \& Unsworth, 1990):

$$
\mathrm{LE}=\frac{\Delta(\mathrm{Rn}-\mathrm{G})+N \mathrm{c}_{\mathrm{p}} \rho^{\left(\mathrm{e}_{\mathrm{s}}-\mathrm{e}_{\mathrm{a}}\right) / \mathrm{r}_{\mathrm{a}}}}{\Delta+\gamma\left(1+\frac{\mathrm{r}_{\mathrm{c}}}{\mathrm{r}_{\mathrm{a}}}\right)}
$$

em que:

$\mathrm{LE}$ - densidade de fluxo de calor latente, $\mathrm{MJ} \mathrm{m}^{-2}$

$\mathrm{Rn}$ - saldo de radiação sobre a cultura, $\mathrm{MJ} \mathrm{m}^{-2}$

$\mathrm{G}$ - densidade de fluxo de calor do solo, $\mathrm{MJ} \mathrm{m}^{-2}$

$\mathrm{e}_{\mathrm{s}}$ - pressão de saturação do vapor d'água, $\mathrm{kPa}$

$\mathrm{e}_{\mathrm{a}}$ - pressão atual de vapor d’água média diária, $\mathrm{kPa}$

$\Delta$ - tangente à curva de pressão de vapor no ponto de temperatura média diária, $\mathrm{kPa}^{\circ} \mathrm{C}^{-1}$

$\gamma$ - coeficiente psicrométrico, $\mathrm{kPa}{ }^{\circ} \mathrm{C}^{-1}$

$\mathrm{r}_{\mathrm{a}}$ - resistência aerodinâmica para difusão de calor e vapor, $\mathrm{s} \mathrm{m}^{-1}$

$r_{c}$ - resistência do dossel vegetativo, $\mathrm{s} \mathrm{m}^{-1}$

$\mathrm{N}$ - fator de conversão de unidade de tempo da resistência do ar com valor de 86400

$\mathrm{C}_{\mathrm{p}}$ - calor específico do ar a pressão constante, $\mathrm{MJ} \mathrm{kg}^{-1} \mathrm{~m}^{-2}$

$\rho$ - densidade do ar, $\mathrm{kg} \mathrm{m}^{-3}$

Obteve-se a ETc dividindo-se LE por $\mathrm{L}$, em que $\mathrm{L}=2,50$ $\left(2,361 \times 10^{-3} \mathrm{~T}\right)$ é o calor latente de evaporação $\left(\mathrm{MJ} \mathrm{kg}^{-1}\right)$. A pressão de saturação de vapor $\mathrm{e}_{\mathrm{s}}$ foi obtida de acordo com a equação de Murray (1967):

$$
\mathrm{e}_{\mathrm{s}}=0,6108 \exp \frac{17,27 \mathrm{~T}}{237,3+\mathrm{T}}
$$

A pressão atual do vapor d’água foi obtida através da expressão:

$$
\mathrm{e}_{\mathrm{a}}=\frac{\mathrm{e}_{\mathrm{s}} \mathrm{UR}}{100}
$$

em que: UR é a umidade relativa do ar.

Obteve-se a inclinação $(\Delta)$ da curva de pressão de saturação do vapor d'água em função da temperatura do ar a partir da equação proposta por Allen et al. (1998):

$$
\Delta=\frac{4098 \mathrm{e}_{\mathrm{s}}}{(\mathrm{T}+237,3)^{2}}
$$

A resistência da cultura ao transporte de calor latente dentro da estufa foi considerada dependente do nível de radiação global, temperatura do ar e umidade do ar, com base em leis exponenciais. Para a cultura do tomate em estufa, os efeitos da radiação na resistência estomática são cruciais e apresentam a seguinte relação (Boulard et al., 1991).

$$
r_{s}=200\left(1+\frac{1}{\exp (0,05(\operatorname{Rg}-50))}\right)
$$

em que:

$\mathrm{r}_{\mathrm{s}}$ - resistência da cultura, $\mathrm{s} \mathrm{m}^{-1}$

$\mathrm{Rg}$ - radiação global média diurna $\left(\mathrm{W} \mathrm{m}^{-2}\right)$ no interior do ambiente protegido.

Obtiveram-se os valores de resistência aerodinâmica através do comprimento médio da folha e velocidade do vento dentro da estufa. De acordo com Boulard \& Wang (2000), tem-se:

$$
r_{a}=220 \frac{d^{0,2}}{{V V_{i}^{0,8}}^{0,8}}
$$

em que:

$r_{a}$ - resistência aerodinâmica, $\mathrm{s} \mathrm{m}^{-1}$

$\mathrm{d}$ - comprimento da folha, $\mathrm{m}$

$\mathrm{VV}_{\mathrm{i}}$ - velocidade do vento dentro da estufa, $\mathrm{m} \mathrm{s}^{-1}$

Os valores da evapotranspiração da cultura obtida pelo método de Penman-Monteith foram comparados com os valores de evapotranspiração obtidos pelo lisímetro; esta comparação também incluiu coeficiente de correlação (v) e o índice de concordância (d) de Wilmott (1985). A partir do balanço hídrico nos lisímetros a evapotranspiração da cultura pode ser obtida através da equação seguinte:

$$
\mathrm{Etc}=\mathrm{P}+\mathrm{I}-\mathrm{D}-\mathrm{R} \pm \Delta \mathrm{A}
$$

em que:

ETc - evapotranspiração da cultura, mm

$\mathrm{P}$ - precipitação, mm

I - lâmina de irrigação, mm

$\mathrm{D}$ - lâmina de drenagem profunda, mm

$\mathrm{R}$ - escoamento superficial, mm

$\Delta \mathrm{A}$ - variação do armazenamento da água do solo na camada de profundidade de zero a $\mathrm{Z}(\mathrm{mm})$, para o intervalo de tempo $(\Delta \mathrm{t})$ considerado no 
balanço; nesta pesquisa, $\mathrm{Z}$ foi igual a $30 \mathrm{~cm}$ e $\Delta \mathrm{t}$ decendial

A variação no armazenamento de água no perfil do solo $(\Delta \mathrm{A})$ foi determinada pela diferença dos valores do conteúdo de água do solo obtidos do perfil nos tempos inicial e final de cada período considerado, expressa pela seguinte equação:

$$
\Delta \mathrm{A}=\left(\theta_{\mathrm{f}}-\theta_{\mathrm{i}}\right) \mathrm{Z}=\mathrm{A}_{\mathrm{f}}-\mathrm{A}_{\mathrm{i}}
$$

em que:

$\theta_{\mathrm{f}}$ - umidade do solo no tempo final, $\mathrm{m}^{3} \mathrm{~m}^{-3}$

$\theta \mathrm{i}$ - umidade do solo no tempo inicial, $\mathrm{m}^{3} \mathrm{~m}^{-3}$

$A_{f}$ e $A_{i}$ - armazenamentos de água no solo no tempo final e inicial, respectivamente

Obteve-se o coeficiente de cultura (Kc) por meio da expressão apresentada por Doorenbos \& Kassam (1979), como:

$$
\mathrm{K}_{\mathrm{c}}=\frac{\mathrm{ET}_{\mathrm{c}}}{\mathrm{ET}_{\mathrm{o}}}
$$

em que:

$\mathrm{ET}_{\mathrm{C}}$ - evapotranspiração da cultura, $\mathrm{mm} \mathrm{d}^{-1}$

$\mathrm{ET}_{\mathrm{o}}$ - evapotranspiração de referência, $\mathrm{mm} \mathrm{d}^{-1}$

A área foliar foi calculada pela seguinte expressão:

$$
\mathrm{AF}=\mathrm{C} \mathrm{L} \mathrm{f}
$$

em que:

$$
\begin{aligned}
& \text { Af - área foliar, } \mathrm{m}^{2} \\
& \mathrm{C} \text { - comprimento, cm } \\
& \mathrm{L} \text { - largura da folha, cm } \\
& \mathrm{f} \text { - fator de correção (fator de forma), o qual foi de- } \\
& \text { terminado pela análise de regressão simples en- } \\
& \text { tre a área de uma amostra de folhas e o produ- } \\
& \text { to de suas dimensões; neste caso, a reta ajustada } \\
& \quad \text { ao conjunto de dados possui uma equação do } \\
& \text { tipo Y }=\text { bx, em que "b” corresponde ao fator “f” }
\end{aligned}
$$

A área das folhas amostradas foi determinada pela digitalização das respectivas imagens que, após serem digitalizadas, foram recortadas, respeitando-se a forma e se realizando a pesagem dessas folhas, obtendo-se então uma relação do peso das folhas com a área da folha. O Índice de área foliar (IAF, $\mathrm{m}^{2} \mathrm{~m}^{-2}$ ) foi calculado por meio da razão área foliar $\left(\mathrm{AF}, \mathrm{m}^{2}\right)$ e área do solo disponível às plantas, $\left(\mathrm{AT}, \mathrm{m}^{2}\right)$, ou seja:

$$
\mathrm{IAF}=\mathrm{AF} \frac{\mathrm{NP}}{\mathrm{AT}}
$$

em que NP é o número de plantas.

\section{RESULTADOS E DISCUSSÃO}

Na fase vegetativa a temperatura média diurna apresentou grandes variações (Figura 1A); entretanto, não superou a temperatura máxima permitida, que é de $30^{\circ} \mathrm{C}$ (Pereira et al., 2000). No período noturno a temperatura variou em

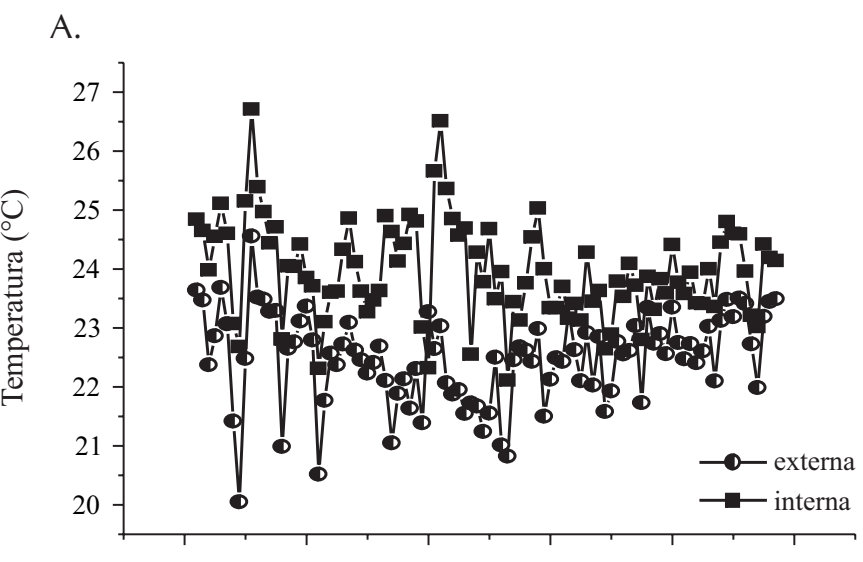

B.
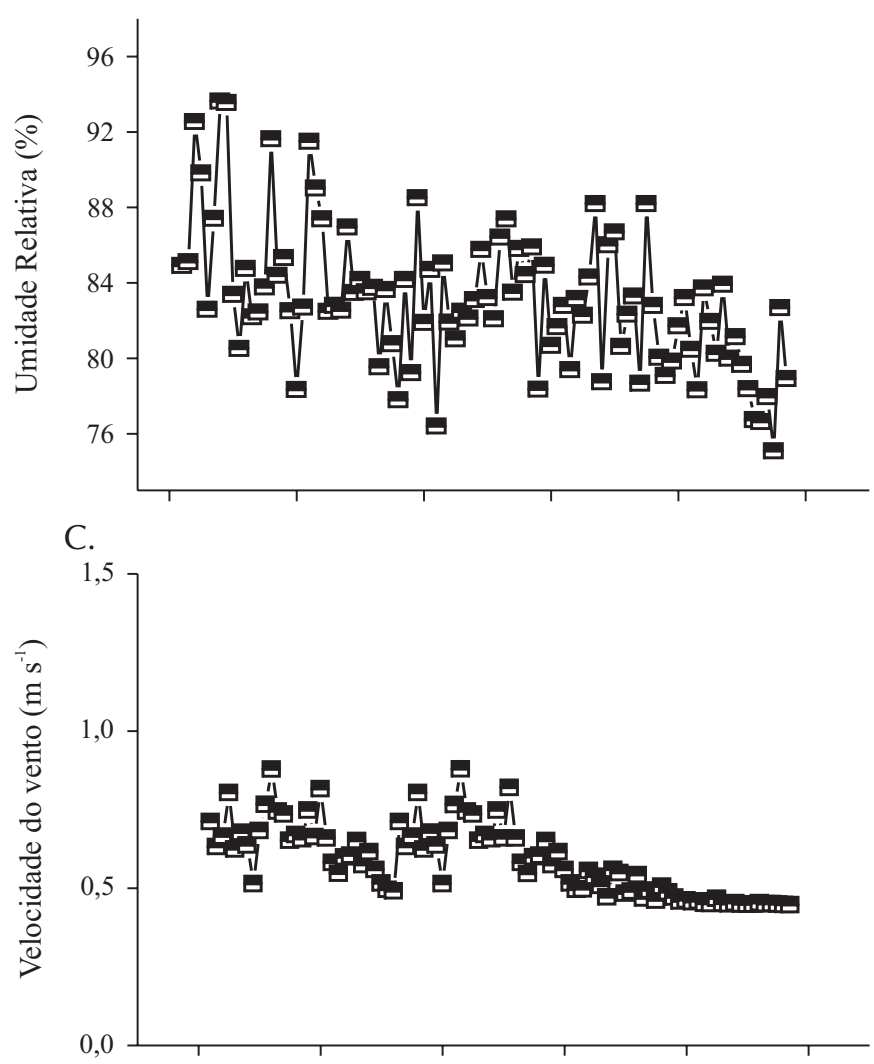

D.

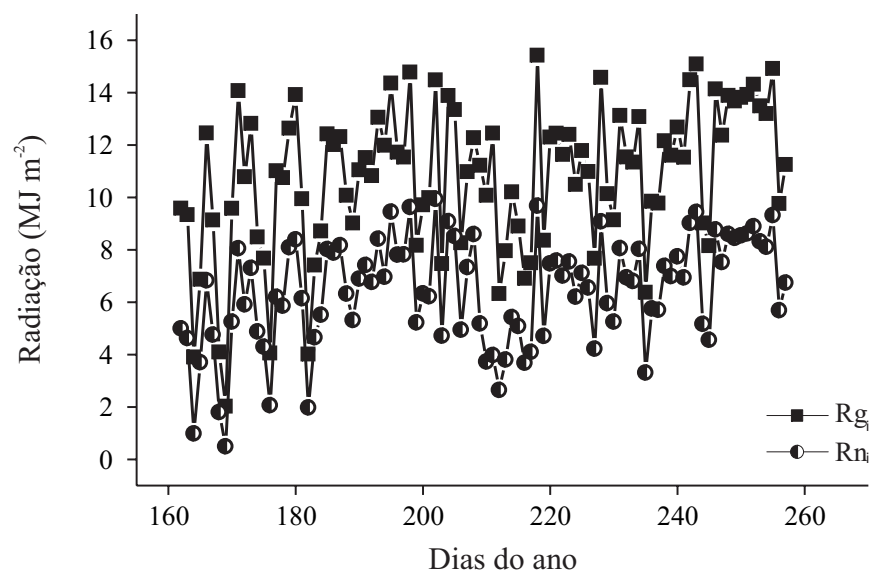

Figura 1. Temperatura do ar (A), umidade relativa do ar (B), velocidade do vento $(C)$, radiação solar global e saldo de radiação (D) no interior da estufa, no período de crescimento e desenvolvimento da cultura 
menor escala apresentando-se, na maioria dos dias, inferior aos valores encontrados no período diurno. Nota-se um valor mais baixo neste período coincidindo com o período de maturação, de $18,5^{\circ} \mathrm{C}$, não afetando os frutos, que suportam até $13^{\circ} \mathrm{C}$, sem perda na qualidade nem no tamanho.

Os valores médios de umidade relativa obtidos no interior da estufa plástica apresentaram uma amplitude menor quando comparados com a umidade relativa fora (URe), justificando um ajuste polinomial de URi $=1,04047$ URe e coeficiente de correlação $\mathrm{R}^{2}=0,93$; encontraram-se diferenças de 5,7\% entre os dois ambientes em termos médios, ao longo do ciclo. A umidade relativa no interior (URi) da estufa apresentou maiores variações nos primeiros vinte dias, com média diária de $80 \%$. Verificou-se que a velocidade do vento dentro do ambiente protegido atingiu o valor máximo de $0,88 \mathrm{~m} \mathrm{~s}^{-1}$ e foi decrescendo até $0,45 \mathrm{~m} \mathrm{~s}^{-1}$

Outra condição meteorológica climática que influencia o ciclo produtivo do tomateiro é a radiação solar, sendo o elemento ambiental que mais contribui para as flutuações de florescimento e formação de frutos. O valor limite de radiação solar diária estabelecido pela FAO e que permite à planta seu pleno desenvolvimento, é de 8,4 $\mathrm{MJ} \mathrm{m}^{-2}$.

O saldo de radiação total encontrado no ambiente protegido foi de $615,53 \mathrm{MJ} \mathrm{m}^{-2}$ representando, em termos energéticos, $60 \%$ da componente da radiação solar global. Durante o período de medições a razão entre o saldo de radiação e a radiação solar global $(\mathrm{Rn} / \mathrm{Rg})$ variou entre 0,65 e 0,24. A equação de regressão obtida $(\mathrm{Rn}=0,60 \mathrm{Rgi})$ teve um coeficiente de determinação de 0,92.

Nota-se que a fração de radiação solar global transformada em energia disponível ao sistema, ou seja, o saldo de radiação (Figura 1D), apresenta distribuição regular e valor médio de $0,5819 \pm 0,08$, alcançando valor máximo de 0,70, ocorrido na fase de frutificação aos 45 dias após o transplantio, sendo que os menores valores ocorreram nos primeiros dias do experimento no ambiente protegido. Ortega-Farías et al. (2004) afirmam que o saldo de radiação (Rn) disponível é a força principal na determinação das perdas de água de uma cobertura vegetal quando a água no solo não é limitante.

A soma das lâminas de irrigação nos períodos floração e frutificação (35-69 DAT) foi de 134,20 mm de água aplicada ao solo e a evapotranspiração do período foi $115,75 \mathrm{~mm}$, com média de $2,89 \mathrm{~mm} \mathrm{~d}^{-1}$. Na fase de maturação dos frutos a lâmina total aplicada foi de 111,69 mm e a evapotranspiração da cultura, de $98,04 \mathrm{~mm}$, com média de 3,27 $\mathrm{mm} \mathrm{d}^{-1}$ (Tabela 1). O maior erro percentual ocorre quando o balanço hídrico do solo é realizado para períodos de um dia, mas quando se tem períodos de uma ou mais semanas, o erro percentual se mantém na faixa de 7,0 a 8,0\% (Silva et al., 2001), razão por que se optou, nesta pesquisa, por fazer o balanço para dez dias, assumindo-se menores erros.

Em relação ao consumo de água na fase de maturação dos frutos (64 DAT) observa-se, neste último período, a ocorrência de maior demanda hídrica, fase em que o saldo de radiação apresentou maiores valores que a fase vegetativa; resultados semelhantes foram encontrados por Ortega-Farías et al. (2001).
Tabela 1. Evapotranspiração da cultura (ETc) do tomate caqui, pelos métodos de Penman-Monteith e do lisímetro

\begin{tabular}{ccc}
\hline $\begin{array}{c}\text { Período } \\
\text { (DAT) }\end{array}$ & $\begin{array}{c}\text { ETc Lisímetro } \\
\left(\mathbf{m m ~ d}^{-1} \mathbf{)}\right.\end{array}$ & $\begin{array}{c}\text { ETc Penman-Monteith } \\
\left(\mathbf{m m ~ d}^{-1}\right)\end{array}$ \\
$34-43$ & 2,4 & 2,5 \\
$44-53$ & 2,6 & 2,5 \\
$54-63$ & 2,8 & 1,8 \\
$64-73$ & 3,7 & 2,3 \\
$74-83$ & 3,0 & 2,1 \\
$84-104$ & 3,2 & 2,5 \\
$105-114$ & 3,7 & 2,9 \\
Média & 3,1 & 2,4 \\
\hline
\end{tabular}

Os valores de ETc encontrados pelo modelo de PenmanMonteith e Lisímetro, acumulados no período de 35 DAT até 121 DAT, foram de 165,46 e 213,79 mm, respectivamente, significando que o modelo de Penman-Monteith subestimou, na média, em $23 \%$ o valor encontrado pelo lisímetro. Abtew \& Obeyssekera (1995) encontraram, em comparações feitas com lisímetro, uma relação satisfatória da equação de Penman-Monteith para estimar a ETr, com 74\% de correlação; por outro lado, Ortega-Farías et al. (2000), afirmam que o método de Penman-Monteith poderia ser mais útil para calcular o consumo de água de tomates submetidos a um stress hídrico temporário.

Observou-se, nos primeiros vinte dias após o transplantio, a ocorrência de uma boa aproximação dos valores estimados pelo modelo de Penman-Monteith, quando comparados com os valores calculados no lisímetro, em que a evapotranspiração média foi de 2,5 e 2,4, respectivamente; a partir daí e apesar do comportamento semelhante dos dois métodos, os valores encontrados pelo método do balanço hídrico foram maiores que os estimados pelo método Penman-Monteith.

No período em que as flores começaram a abrir e surgiram os primeiros frutos, fase reprodutiva (45-64 DAT), deuse um aumento da ETc, observado pelo método do balanço hídrico mas não pelo modelo Penman-Monteith, que subestimou os valores de ETc em até 38\% porém entre os 66-116 DAT os valores medidos no lisímetro e os estimados pela equação de Penman-Monteith apresentam aumento e a diferença entre eles decresce gradualmente, atingindo $21 \%$, fase esta coincidente com o aparecimento dos primeiros frutos maduros (fase de maturação), com o que se espera maior consumo de água pela cultura. Esse fato tem sido explicado, segundo alguns autores (Ortega-Farías et al., 2004; Valdés et al., 2004), em função do modelo de Penman-Monteith ser impróprio para culturas na fase inicial de crescimento, haja vista que o índice de área foliar (IAF) é muito pequeno (Figura 2), embora o modelo seja preciso quando o IAF é maior que 2 .

O modelo subestima os valores de evapotranspiração para um baixo índice de área foliar, mas, se for considerado o $r_{c}$ variável, este problema desaparecerá. Na determinação da evapotranspiração para culturas com IAF pequeno e solo com déficit hídrico temporário, o modelo de Penman-Monteith requer uma parametrização adequada de $r_{c}$ que inclui as interações não-lineares entre condições atmosféricas, solo, planta e conteúdo de água no solo. 


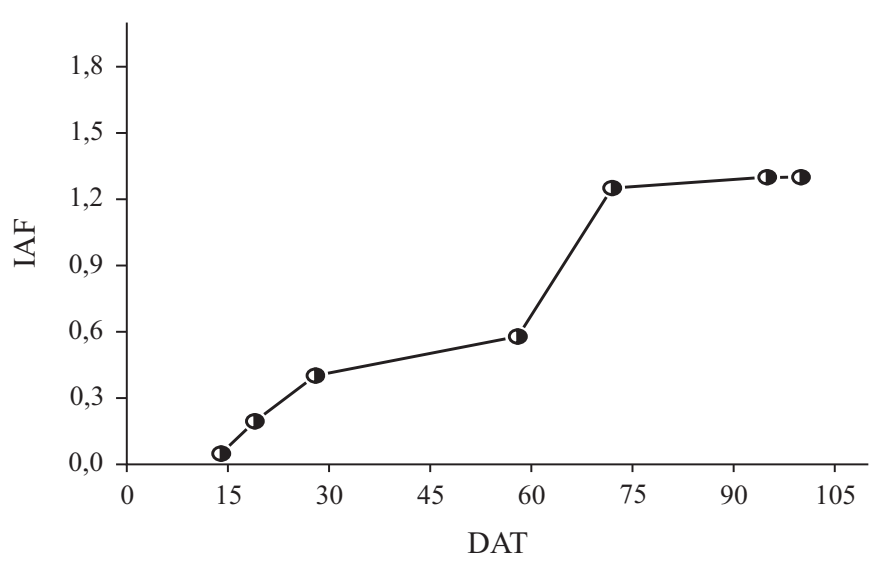

Figura 2. Índice de área foliar durante o crescimento e desenvolvimento da cultura

A equação de Penman-Monteith pode simular, de hora em hora e diariamente, a evapotranspiração total da cultura do tomate cultivada em estufa (Valdés et al., 2004); entretanto, os valores encontrados neste trabalho pelo método de Penman-Monteith ficaram abaixo dos indicados pelo método do balanço hídrico, durante todo o ciclo da cultura, com exceção da primeira semana. O método de balanço hídrico ocasiona uma superestimativa da evapotranspiração da cultura, segundo Lima et al. (1994), para determinação da evapotranspiração máxima do tomateiro, nos subperíodos mais frios.

Comparando-se a evapotranspiração da cultura medida no lisímetro e estimada pelo método Penman-Monteith no interior da estufa (Figura 3), obtém-se Y $=0,75 \mathrm{X}$, observando-se que os valores dos coeficientes $\mathrm{r}$ e $\mathrm{d}$ foram de $0,84 \mathrm{e}$ 0,74 , respectivamente, resultando em um valor de c $=0,62$ considerado de desempenho mediano; isto demonstra que ocorre uma subestimativa predominante do método de Penman-Monteith.

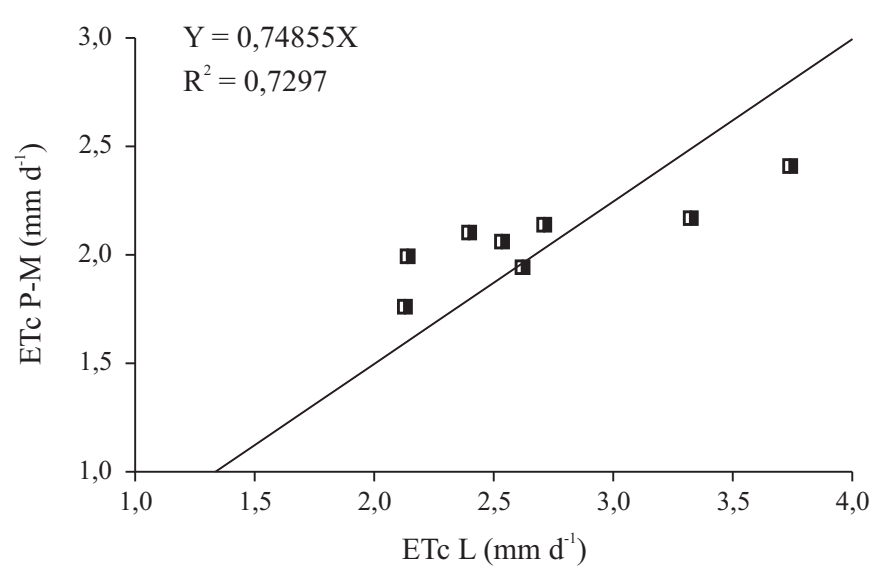

Figura 3. Correlação entre evapotranspiração da cultura medida no lisímetro (ETC-L) e estimada pelo método Penman-Monteith (ETC-PM) no interior da estufa

Os valores do Kc para os diferentes estágios de desenvolvimento estimado pelo método de Penman-Monteith (Kc-PM) foram de 0,94, 0,74 e 0,53 para fase vegetativa, reprodutiva e maturação, respectivamente. A fase vegetativa foi conside- rada a partir de 20 dias após o transplantio; o Kc médio durante todo o ciclo da cultura ficou em torno de 0,66 \pm 0,37 com evapotranspiração da cultura máxima de $2,8 \mathrm{~mm}$ e mínima $0,37 \mathrm{~mm}$ e um erro de $0,038 \mathrm{~mm}$; na fase reprodutiva o Kc foi maior que na fase vegetativa em virtude do maior consumo da planta nesta fase (Figura 4).

Os coeficientes de cultivo nas fases vegetativa, reprodutiva e maturação do tomate caqui, utilizando-se a ETc estimada pelo método de Penman-Monteith cultivado em estufa, foram 0,$7 ; 0,74$ e 0,53 , respectivamente.
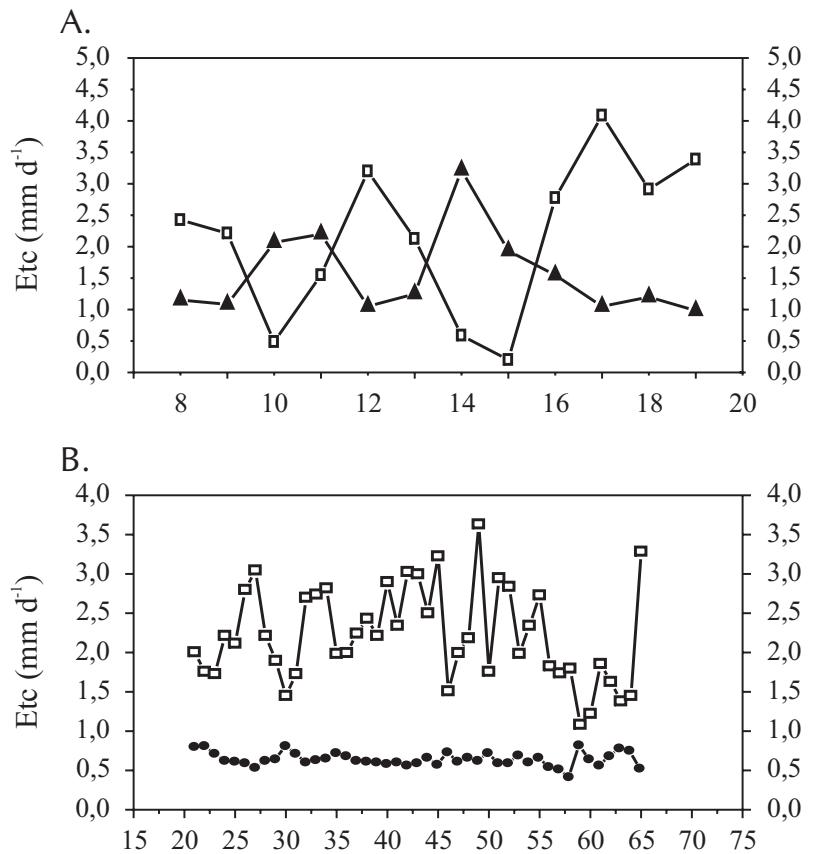

C.

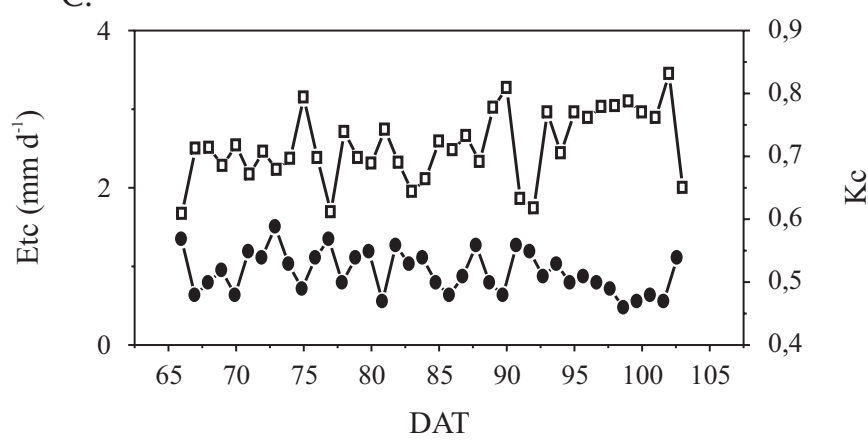

Figura 4. Evapotranspiração e Kc da cultura em diferentes estágios de crescimento em estufa pelo método Penman-Monteith (ETc-PM)

Castilla et al. (1990) encontraram, em estudos conduzidos durante três anos com tomate em lisímetro instalado no interior de casa de vegetação, valores de ET, para a cultivar vermone, da ordem de 288 a 356 mm, e o coeficiente de cultura atingiu o valor 1,2 , decrescendo para 0,8 ao final do ciclo.

Observa-se, na Figura 4, que o Kc apresentou grande variação chegando a um valor médio de 0,95 no período vegetativo; este valor está abaixo dos sugeridos pela FAO, isto é, de 0,6, 1,4 e 1,0 para as fases inicial, intermediária e final, respectivamente; a partir do 30 DAT o Kc da cultura se estabiliza 
e o valor permanece até 60 DAT em média 0,7, finalizando no período de maturação com o valor de Kc de 0,65.

Esses valores, por sua vez, são inferiores aos encontrados por Lima et al. (1994) que, trabalhando com tomateiro cultivar IPA-5, observaram grande variação na fase fenológica quando comparados com os das outras regiões do mundo; situando-se dentro dos limites estabelecidos pela FAO (Doorenbos \& Kassam, 1979), nas diferentes fases da cultura, Kc estaria próximo de 0,45 no período inicial da cultura, devido principalmente ao baixo índice de área foliar e, no final do período, em razão do declínio da cultura, abscisão das folhas e fim da colheita; entretanto, Amayreh \& AlAbed (2005), encontraram valores médios de Kc do tomate de 0,69 para todo o ciclo, sendo 0,8 para o estágio reprodutivo e 0,45 para o estágio de maturação; considerando-se os valores da FAO, constatou-se que referidos resultados foram 33 a $40 \%$ menores.

\section{CONCLUSÕES}

1. Em condições de ambiente protegido, os valores de ETc encontrados pelos métodos de Penman-Monteith e do lisímetro, acumulados no período de crescimento até a maturação dos frutos, foram de 165,46 e 213,79 mm, respectivamente, representando, na média, uma subestimativa do modelo de Penman-Monteith em 23\% do valor encontrado pelo lisímetro.

2. O Kc médio encontrado foi de 0,65 para as condições do experimento.

\section{LITERATURA CITADA}

Abtew, W.; Obeysekera, J. Lysimeter study of evapotranspiration of cattails and comparison of three estimation methods. Transactions of the ASAE, v.38, n.1, p.121- 129, 1995.

Allen, R. G.; Pereira, L. S.; Raes, D.; Smith, M. Crop evapotranspiration: Guidelines for computing crop water requirements. Rome: FAO, 1998. 300p. (Irrigation and Drainage, 56).

Amayreh, J.; Al-Abed, N. Developing crop coefficients for fieldgrown tomato (Lycopersicon esculentum Mill.) under drip irrigation with black plastic mulch. Agricultural Water Management, v.73, n.3, p.247-254, 2005.

Blanco, F. F.; Folegatti, M. V. New method for estimating the leaf area index of cucmber and tomato plants. Horticultura Brasileira, v.21, n.4, p.666-669, 2003.

Boulard, T.; Bailey, A.; Mermier, M.; Vilette, F. Measures et modelisation de la resistance stomatique foliaire et de la transpiration d'un couvert de tomate de serre. Agronomie, n.11, p.259-274, 1991.

Boulard, T.; Wang, S. Greenhouse crop transpiration simulation from external climate conditions. Agricultural and Forest Meteorology, v.100, n.1, p.25-34, 2000.

Buriol, G. A.; Streck, N. A.; Schneider, F. M. Modificação ambiental causada por túneis baixos de polietileno transparente perfurado cultivado com alface. Ciência Rural, v.23, n.3, p.261-266, 1993.
Carrijo, O. A.; Vidal, M. C.; Reis, N. V. B.; Souza, R. B.; Makisima, N. Produtividade do tomateiro em diferentes substratos e modelos de casas de vegetação. Horticultura Brasileira, v.22, n.1, p.5-9, 2004.

Castilla, N.; Elias, F.; Fereres, E. Evapotranspiracion de cultivos horticolas en invernadero en Almeria. Investigacion Agraria: Produccion y Proteccion Vegetal, v.5, p.117-125, 1990.

Cunha, A. R.; Escobedo, J. F. Alterações micrometeorológicas causadas pela estufa plástica e seus efeitos no crescimento e produção da cultura de pimentão. Revista Brasileira de Agrometeorologia, v.11, n.1, p.15-26, 2003.

Cunha, A. R.; Escobedo, J. F.; Klosowski, E. S. Efeito do fluxo de calor latente pelo balanço de energia em cultivo protegido de pimentão. Pesquisa Agropecuária Brasileira, v.37, n.6, p.735-743, 2002.

Dalsasso, L. C. M.; Heldwein, A. B.; Buriol, G. A.; Scheneider, F. M.; Streck, N. A.; Dalmago, G. A. Consumo de água do tomateiro tipo salada em estufa plástica, Revista Brasileira de Agrometeorologia, v.5, n.1, p.61-67, 1997.

Doorenbos, J.; Kassam, A. H. Efectos del agua el rendimiento de los cultivos. Roma: FAO. 1979. 194 p. (Irrigation and Drainage Paper, 24).

Doorenbos, J.; Pruitt, W. O. Las necesidades de agua de los cultivos. Roma: FAO, 1976. 194p. (Estúdio FAO Riego y Drenage, 24).

Farias, J. R. B.; Bergamashi, H.; Martins, S. R.; Berlato, M. A. Efeito da cobertura plástica da estufa sobre a radiação solar. Revista Brasileira de Agrometeorologia, v.1, n.1, p.31-36, 1993.

Fayad. J. A.; Fontes, P. C. R.; Cardoso, A. A.; Finger, F. L.; Ferreira, F. A. Horticultura Brasileira, v.19, n.3, p.9-13, 2001.

Heldewin, A. B.; Streck, L.; Schneider, F. M.; Grimm, E. L.; Nied, A. H.; Tazzo, I. F. Modelos para estimativa da evapotranspiração máxima da abóbora italiana em estufa plástica. Revista Brasileira de Agrometeorologia, v.12, n.1, p.75-86, 2004.

Joillete , O.; Bailey, B. J. The efects of climate on tomato transpiration in geenhouse: messurements and models comparison. Agricultural and Forest Meteorology, v.58, p.43-62, 1992.

Kirda, C.; Bay, N. T.; Derici, R.; Dasgan, H. Nitrogen fertilizer recovery and yield response of greenhouse grown and fertigated tomato to root - zone soil water tension. Journal Agricultural For, v.27, p.323-328, 2003.

Lima, M. G.; Leal, F. R.; Setubal, J. W. Evapotranspiração máxima e coeficiente de cultura para o tomateiro em Teresina - PI. Revista Brasileira de Agrometeorologia, v.2, n.1, p.29-32, 1994.

Luz, J. M. Q.; Shinzato, A. V.; Silva, M. A. D. Comparação dos sistemas de produção de tomate convencional e orgânico em cultivo protegido. Bioscience Journal, v.23, n.2, p.7-15, 2007.

Miranda, F. R.; Oliveira, J. J. G.; Souza, F. Evapotranspiração máxima e coeficiente de cultivo para a cultura da melancia. Revista Ciência Agronômica, v.35, n.1, p.36-43, 2004.

Monteith, J. L.; Unsworth, M. H. Principles of environmental physics. 2.ed. London: Eward Arnold, 1990. 291p.

Murray, F. W. On the computation of saturation vapor pressure. Journal of Applied Meteorology, v.6, p.203-204. 1967.

Oliveira, M. R. V. O emprego de casas de vegetação no Brasil: Vantagens e desvantagens. Pesquisa Agropecuária Brasileira, v.30, n.8, p.1049-60, 1995. 
Orgaz, F.; Fernández, M. D.; Bonachela, S.; Gallardo, M.; Fereres, E.Agricultural Water Managment, v.72 , p.81-96, 2005.

Ortega-Farías, S.; Calderon R.; Acevedo, C.; Fuentes, S. Estimation de la evapotranspiracion real diaria de un cultivo de tomates usando la equacion de Penmam-Monteith. Ciencia Investigaçao Agraria, v.27, n.2, p.91-96, 2000.

Ortega-Farías, S.; Calderon R.; Martelli, N.; Antonioletti, R. Evaluacion de un modelo para estimar la radiacion neta sobre un cultivo de tomate industrial. Agricultura Técnica, v.61, n.14, p.41-49, 2004.

Ortega-Farías, S.; Cuenca, R. H. Daytime variation of sensible heat flux estimated by the bulk aerodynamic method over a grass canopy. Agricultural and Forest Meteorology, v.81, n.12, p.131-143. 1996.

Ortega-Farías, S.; Cuenca, R. H. Estimation of crop evapotranspiration by using the Penman-Monteith method with a variable canopy resistance. Water Resources Engineering' 98, edited by Abt, S. R.; Young-Pezeshk, J.; Watson, C. C. American Society of Civil Engineers, v.2, p.1806-1811, 1998.

Ortega-Farías, S. Márquez J.; Valdés, H.; Paillán, H. Effect of four different levels of water application on yield and quality of greenhouse tomatoes (Lycopersicon esculentum Mill. cv. FA-144) produced in autumn. Agricultura Técnica, v.61, n.4, p.479-487, 2001.

Pereira, C.; Marchi, G.; Silva, E. C. Produção de Tomate-caqui em Estufa. Série extensão. Lavras: Universidade Federal de Lavras, 2000. 26p.
Rana, G.; Katerji, N. Measurement and estimation o factual evapotranspiration in the fild under Mediterranean climate: A review. European Journal of Agronomy, v.13, p.125-153, 2000.

Silva, V. P. R.; Azevedo, P. V.; Silva, B. B. da; Bassoi, L. H.; Texeira, A. H. C.; Soares, J. M.; Silva, J. A. M. Estimativa da evapotranspiração da mangueira com base no balanço hídrico do solo. Revista Brasileira de Engenharia Agrícola e Ambiental, v.5, n.3, p.456-462, 2001.

Souza, D. K. S.; Lopes, M. J. A.; Nascimento Filho, M. F. Efeito da tela plástica na temperatura do solo e radiação solar na cultura do pimentão. In: Congresso Brasileiro de Agrometeorologia, 9, 1997, Campina Grande. Anais... Campina Grande: Sociedade Brasileira de Agrometeorologia. 1997. p.339-41.

Souza, J. L. de ; Nicácio, R. M.; Moura, M. A. L .Global solar radiation measurements in Maceió, Brazil. Renew Energy, v.30, 1205-1220, 2005.

Valandro, J.; Andriolo, L.; Buriol, G. A. Dispositivo lisimétrico simples para determinar a transpiração das hortaliças cultivadas fora do solo. Revista Brasileira de Agrometeorologia, v.7, n.2, p.189-193, 1999.

Valdés, H.; Ortega-Farías, S.; Argote, M.; Leyton, B.; Olioso, A.; Paillán, H. Estimation of evapotranspiration over a greenhouse tomato crop using the Penman-Monteith equation. Acta Horticulturae, v.664, p.477-482, 2004.

Wilmott, J. D. Estatistics for the evaluation and comparision of model. Journal of geophysical Research, v.50, n.5, p.89959005, 1985. 